\title{
COHERENT PAIRS OF EXTENSIONS OF ASSOCIATIVE ALGEBRAS
}

\author{
N. RAMABHADRAN
}

Let $K$ be a commutative ring with identity element and let $\Lambda_{i}, i=1,2$ be two $K$-projective associative algebras with identity element such that the map $k \rightarrow k \cdot 1$ of $K$ into $\Lambda_{i}$ is a monomorphism of $K$ onto a $K$-direct summand of $\Lambda_{i}$, $i=1,2$. Let $\Lambda=\Lambda_{1} \otimes \Lambda_{2}$ and $A$ be a two sided $\left(\Lambda_{1}, \Lambda_{2}\right)$-bimodule. Let $(\Sigma): 0 \longrightarrow A \stackrel{\beta}{\rightarrow} \Gamma \stackrel{a}{\longrightarrow} \Lambda \longrightarrow 0$ be an extension over $\Lambda$ with kernel $A$ (abelian). This gives rise to a pair $\left(\Sigma_{1}\right),\left(\Sigma_{2}\right)$ of extensions $\left(\Sigma_{i}\right): 0 \rightarrow A \rightarrow \alpha^{-1}\left(\Lambda_{i}\right) \rightarrow \Lambda_{i} \rightarrow 0$ over $\Lambda_{i}$ with kernel $A, i=1,2$. The object of this paper is to give a characterization of pairs of extensions over $A_{1}$ and $\Lambda_{2}$ respectively, with kernel $A$ which arise in this way from an extension over $\Lambda_{1} \otimes \Lambda_{2}$ with kernel $A$. This leads to the notion of coherent pairs of extensions (Def. 3.8). The corresponding problem for groups has been treated by F. Haimo and S. Maclane [2]. We define bicohomology groups $H^{n}\left(\Lambda_{1}, \Lambda_{2} ; A\right)$ of the pair $\left(\Lambda_{1}, \Lambda_{2}\right)$ and show (Prop. 3.14) that the set $\mathscr{C}\left(\Lambda_{1}, \Lambda_{2} ; A\right)$ of all coherent pairs of equivalence classes of extensions forms a $K$-module which is a homomorphic image of $H^{2}\left(\Lambda_{1}, \Lambda_{2} ; A\right)$. The kernel of this homomorphism is also determined (Prop. 3.16). The analogous problem for Lie algebras has also been treated by us and will appear elsewhere. We have followed the notation and terminology, as in [1] and [2]. All tensor products are over $K$.

\section{Bicohomology of a pair of associative algebras}

Let $K$ be a commutative ring with identity element 1 and $\Lambda_{1}$ and $\Lambda_{2}$ be two $K$-projective associative algebras with identity element. Further, let the map $k \rightarrow k \cdot 1$ of $K$ into $\Lambda_{i}$ be monomorphism of $K$ onto a $K$-direct summand of $\Lambda_{i}$, $i=1,2$. Let $A$ be a two sided $\left(\Lambda_{1}, \Lambda_{2}\right)$-bimodule i.e. $A$ is a two sided $\Lambda_{i}$-module, $i=1,2$ the operators from $\Lambda_{1}$ commuting with those from $\Lambda_{2}$. Let $S\left(\Lambda_{i}\right)=$ $\sum_{n \geq 0} S_{n}\left(\Lambda_{i}\right)$ be the standard complex [1] of the associative algebra $\Lambda_{i}$ where

Received June 15, 1964. 


$$
S_{n}\left(\Lambda_{i}\right)=\Lambda_{i}^{e} \otimes \widetilde{S}_{n}\left(\Lambda_{i}\right), \widetilde{S}_{0}\left(\Lambda_{i}\right)=K
$$

and $\widetilde{S}_{n}\left(A_{i}\right)$ is the tensor product (over $K$ ) of the $K$-module $A_{i}$ taken $n$-times, $n>0, \Lambda_{i}^{e}$ being the enveloping algebra $\Lambda_{i} \otimes \Lambda_{i}^{*}$ of $\Lambda_{i}, i=1,2 . \quad$ It is known [1] (Since $\Lambda_{i}$ is $K$-projective) that $S\left(\Lambda_{i}\right)$ is a $\Lambda_{i}^{e}$-projective resolution of $\Lambda_{i}$. Let $\mathscr{L}^{n}\left(\Lambda_{i}, A\right)=\operatorname{Hom}_{K}\left(\widetilde{S}_{n}\left(\Lambda_{i}\right), A\right), n \geq 0$ and $\mathscr{L}^{n, m}\left(\Lambda_{1}, \Lambda_{2} ; A\right)=\operatorname{Hom}_{K}\left(\widetilde{S}_{n}\left(\Lambda_{1}\right) \otimes\right.$ $\left.\widetilde{S}_{m}\left(\Lambda_{2}\right), A\right), n \geq 0, m \geq 0$. Then the $K$-module

$$
\mathscr{L}\left(\Lambda_{i}, A\right)=\sum_{n \geq 0} \mathscr{L}^{n}\left(\Lambda_{i}, A\right)
$$

is a cochain complex [1] the coboundary operator $\delta_{i}$ being given by

$$
\begin{aligned}
\left(\delta_{i} f\right)\left(\lambda_{1}^{(i)}, \ldots, \lambda_{n+1}^{(i)}\right)=\lambda_{1}^{(i)} f\left(\lambda_{2}^{(i)}, \ldots, \lambda_{n+1}^{(i)}\right) & \\
& +\sum_{0<t<n+1}(-1)^{t} f\left(\lambda_{1}^{(i)}, \ldots, \lambda_{t}^{(i)} \cdot \lambda_{t+1}^{(i)}, \ldots, \lambda_{n+1}^{(i)}\right) \\
& +(-1)^{n+1} f\left(\lambda_{1}^{(i)}, \ldots, \lambda_{n}^{(i)}\right) \cdot \lambda_{n+1}^{(i)}
\end{aligned}
$$

for $f \in \mathscr{L}^{n}\left(\Lambda_{i}, A\right)$ and $\lambda_{1}^{(i)}, \ldots, \lambda_{n+1}^{(i)} \in \Lambda_{i}$. The $K$-modules $\mathscr{L}^{n}\left(\Lambda_{i}, A\right)$ and $\mathscr{L}^{n, m}\left(A_{1}, \Lambda_{2} ; A\right)$ can be considered as two sided $\left(A_{1}, A_{2}\right)$-bimodules through $A$ and, as usual, we can identify $\mathscr{L}^{n, m}\left(\Lambda_{1}, \Lambda_{2} ; A\right)$ with $\mathscr{L}^{n}\left(\Lambda_{1}, \mathscr{L}^{m}\left(\Lambda_{2}, A\right)\right)$ and $\mathscr{L}^{m}\left(\Lambda_{2}, \mathscr{L}^{n}\left(\Lambda_{1}, A\right)\right)$. Let

$$
\mathscr{L}^{n}\left(\Lambda_{1}, \Lambda_{2} ; A\right)=\sum_{0 \leq r \leq n} \mathscr{L}^{r, n-r}\left(\Lambda_{1}, \Lambda_{2} ; A\right), n \geq 0 .
$$

We define a map $\delta$ from $\mathscr{L}^{n}\left(\Lambda_{1}, \Lambda_{2} ; A\right)$ into $\mathscr{L}^{n+1}\left(\Lambda_{1}, A_{2} ; A\right)$ (which is a $K$-homomorphism) as follows. Let $f \in \mathscr{L}^{n}\left(\Lambda_{1}, A_{2} ; A\right), f=f_{n, 0}+\cdots+f_{0, n}$ $f_{r, n-r} \in \mathscr{L}^{r, n-r}\left(\Lambda_{1}, \Lambda_{2} ; A\right)$. Let $\delta f=f^{\prime}=f_{n+1,0}^{\prime}+\cdots+f_{0, n+1}^{\prime}$ where

$$
f_{r, n+1-r}^{\prime}=\delta_{1} f_{r-1, n-r+1}+(-1)^{r} \delta_{2} f_{r, n-r} .
$$

Since $\delta_{1}$ and $\delta_{2}$ commute and $\delta_{i}^{2}=0, i=1,2$ it follows that $\delta^{2}=0$. Thus the graded $K$-module $\mathscr{L}\left(A_{1}, \Lambda_{2} ; A\right)=\sum_{n \geq_{0}} \mathscr{L}^{n}\left(A_{1}, A_{2} ; A\right)$ is a cochain complex with $\delta$ as coboundary operator. Elements of $\mathscr{L}^{n}\left(\Lambda_{1}, \Lambda_{2} ; A\right)$ are called $n$ bicochains of the pair $\left(\Lambda_{1}, \Lambda_{2}\right)$ with values in $A$, those of $Z^{n}\left(\Lambda_{1}, A_{2} ; A\right)=$ Kernel $\delta, \delta: \mathscr{L}^{n} \rightarrow \mathscr{L}^{n+1}$ are called $n$-bicocycles and those of $B^{n}\left(\Lambda_{1}, \Lambda_{2} ; A\right)$ $=$ image of $\delta, \delta: \mathscr{L}^{n-1} \rightarrow \mathscr{L}^{n}, n \geq 1$ are called $n$-bicoboundaries. Further we define $B^{0}\left(\Lambda_{1}, A_{2} ; A\right)$ to be 0 . Since $\delta^{2}=0$ we have the bicohomology module $H^{n}\left(\Lambda_{1}, \Lambda_{2} ; A\right)=Z^{n}\left(\Lambda_{1}, \Lambda_{2} ; A\right) / B^{n}\left(\Lambda_{1}, \Lambda_{2} ; A\right), n \geq 0$. Let $\Lambda=\Lambda_{1} \otimes \Lambda_{2}$. Then $S(A)$ is $A^{e}$-projective resolution of $A$. Since $A_{i}$ is $K$-projective, $i=1,2$, it follows [1] that, the $\Lambda_{1}^{e} \otimes A_{2}^{e}\left(=A^{e}\right)$-module

$$
S\left(A_{1}\right) \otimes S\left(A_{2}\right)=\sum_{n \geq 0} S_{r}\left(A_{1}, \Lambda_{2}\right)
$$


where

$$
S_{r}\left(\Lambda_{1}, \Lambda_{2}\right)=\sum_{0 \leq r \leq n} S_{r}\left(\Lambda_{1}\right) \otimes S_{n-r}\left(\Lambda_{2}\right)
$$

considered as tensor product of the complexes $S\left(A_{1}\right)$ and $S\left(A_{2}\right)$, is a $A^{e}$-projective resolution of $\Lambda$. Thus $H^{n}(\Lambda, A)$, the $n^{\text {th }}$ cohomology module of the $K$-algebra $\Lambda$, can also be considered as the $n^{t \bar{n}}$ derived groups of the complex $\sum_{n \geq 0} \mathrm{Hom}_{\Lambda^{a}}$ $\left(S_{n}\left(\Lambda_{1}, A_{2}\right), A\right)$. Comparing the coboundary operators in this complex and in $\mathscr{L}\left(\Lambda_{1}, \Lambda_{2} ; A\right)$ we have

Proposition 1.1. $H^{n}\left(\Lambda_{1}, \Lambda_{2} ; A\right)$ is isomorphic to $H^{n}(A, A)$ as K-modules, $n \geq 0$.

Let $N\left(A_{i}\right), i=1,2$ and $N(\Lambda)$ denote the normalized standard complexes [1] of the $K$-algebras $\Lambda_{i}$ and $\Lambda$ respectively. $\quad N\left(\Lambda_{i}\right)=\sum_{n \geq 0} N_{n}\left(\Lambda_{i}\right), N_{n}\left(\Lambda_{i}\right)=\Lambda_{i}^{e} \otimes \tilde{N}_{n}\left(\Lambda_{i}\right)$ where $\tilde{N}_{0}\left(\Lambda_{i}\right)=K$ and $\tilde{N}_{n}\left(A_{i}\right)$ is the tensor product (over $K$ ) of the $K$ module $\Lambda_{i}^{\prime}=$ cokernel $\left(K \rightarrow \Lambda_{i}\right)$, taken $n$-times, $n>0$ (Because of our assumption on $\Lambda_{i}$ and $K$, this cokernel is a $K$-direct summand of $\Lambda_{i}$ ). It follows that $N\left(\Lambda_{i}\right)$ (resp. $N(\Lambda)$ ) is a $\Lambda_{i}^{e}$-(resp. $\Lambda^{e}$-) projective resolution of $\Lambda_{i}$ (resp. $\Lambda$ ). Let $N^{n}\left(\Lambda_{i}, A\right)=\operatorname{Hom}_{K}\left(\tilde{N}_{n}\left(\Lambda_{i}\right), A\right)$. Thus $N^{n}\left(A_{i}, A\right)$ can be identified with the submodule of $\mathscr{L}^{n}\left(\Lambda_{i}, A\right)$ consisting of those elements $f$ which take the value 0 if any one of the variables $\lambda_{i}$ is 1 . Such cochains are called normalized cochains. Then $N\left(\Lambda_{i}, A\right)=\sum_{n \geq 0} N^{n}\left(\Lambda_{i}, A\right)$ is a cochain complex with the coboundary operator, denoted again by $\delta_{i}$, given by the formula (1). Similarly $N(\Lambda, A)=\sum_{n \geq 0} N^{n}(\Lambda, A)$ is a cochain complex (normalized). Thus $H^{n}\left({ }_{1} 1_{i}, A\right)$, $i=1,2$ (resp. $H^{n}(A, A)$ ) can also be considered as the $n^{\text {th }}$ derived groups of $N\left(A_{i}, A\right)$ (resp. $N(A, A)$ ). Let $\xi$ be the map from $N(\Lambda)$ into $N\left(A_{1}\right) \otimes N\left(A_{2}\right)$ [1] defined by

$$
\begin{aligned}
& \xi\left(\left(\lambda_{0}^{(1)} \otimes \lambda_{0}^{(2)}\right)\left[\lambda_{1}^{(1)} \otimes \lambda_{1}^{(2)}, \ldots, \lambda_{n}^{(1)} \otimes \lambda_{n}^{(2)}\right]\left(\mu_{0}^{(1)} \otimes \mu_{0}^{(2)}\right)\right) \\
= & \sum_{0=r=n} \lambda_{0}^{(1)}\left[\lambda_{1}^{(1)}, \ldots, \lambda_{r}^{(1)}\right] \lambda_{r+1}^{(1)} \cdots \lambda_{n}^{(1)} \cdot \mu_{0}^{(1)} \otimes \lambda_{0}^{(2)} \cdots \lambda_{r}^{(2)}\left[\lambda_{r+1}^{(2)}, \ldots, \lambda_{n}^{(2)}\right] \mu_{0}^{(2)}
\end{aligned}
$$

for $n \geq 0$, and

$$
\lambda_{0}^{(i)}, \ldots, \lambda_{n}^{(i)}, \mu_{0}^{(i)} \in \Lambda_{i}, i=1,2
$$

Then it is known [1] that $\xi$ is a 'map' over the identity of $\Lambda$. $\xi$ gives rise to a $K$-homomorphism $\xi^{*}$ from $\left.\operatorname{Hom}_{\Lambda_{1}{ }^{e} \otimes \Lambda_{2} e} e \sum_{0 \leq r=n} N_{r}\left(\Lambda_{1}\right) \otimes N_{n-r}\left(\Lambda_{2}\right), A\right)$ into $\operatorname{Hom}_{\Lambda^{e}}\left(N_{n}(A), A\right)$ for $n \geq 0$. In particular for $n=2$ we have the following formula 


$$
\begin{aligned}
& \xi^{*}\left(\omega_{1}+r+\omega_{2}\right)\left(\lambda_{1}^{(1)} \otimes \lambda_{1}^{(2)}, \lambda_{2}^{(1)} \otimes \lambda_{2}^{(2)}\right) \\
= & \omega_{1}\left(\lambda_{1}^{(1)}, \lambda_{2}^{(1)}\right) \cdot \lambda_{1}^{(2)} \cdot \lambda_{2}^{(2)}+\lambda_{1}^{(2)} r\left(\lambda_{1}^{(1)}, \lambda_{2}^{(2)}\right) \cdot \lambda_{2}^{(1)}+\lambda_{1}^{(1)} \cdot \lambda_{2}^{(1)} \omega_{2}\left(\lambda_{1}^{(2)}, \lambda_{2}^{(2)}\right) .
\end{aligned}
$$

Where $\omega_{1} \in \operatorname{Hom}_{\Lambda_{1}^{e} \otimes \Lambda_{2} e}\left(N_{2}\left(\Lambda_{1}\right) \otimes \Lambda_{2}^{e}, A\right)=\operatorname{Hom}_{K}\left(\tilde{N}_{2}\left(\Lambda_{1}\right), A\right)$

$$
r \in \operatorname{Hom}_{\Lambda_{1} e \otimes \Lambda_{2}^{e}}\left(N_{1}\left(\Lambda_{1}\right) \otimes N_{1}\left(\Lambda_{2}\right), A\right)=\operatorname{Hom}_{K}\left(\tilde{N}_{1}\left(\Lambda_{1}\right) \otimes \tilde{N}_{1}\left(A_{2}\right), A\right)
$$

and

$$
\omega_{2} \in \operatorname{Hom}_{\Lambda_{1}^{e} \otimes \Lambda_{2}^{e}}\left(\Lambda_{1}^{e} \otimes N_{2}\left(\Lambda_{2}\right), A\right)=\operatorname{Hom}_{K}\left(\tilde{N}_{2}\left(\Lambda_{2}\right), A\right) .
$$

If $\omega=\xi^{*}\left(\omega_{1}+r+\omega_{2}\right)$ then $\omega$ is a 2 -cochain (normalized) of $A$ with values in $A$ and $\omega$ is a 2-cocycle if and only if (i) $\omega_{i}$ is a 2-cocycle and (ii) $\delta_{i} \omega_{j}=(-1)^{j} \delta_{j} r$, $i \neq j, i=1,2$ (The formula (4) will be used later in Prop. 3.10).

\section{Stability Lie algebra}

Let $\Gamma$ be an associative $K$-algebra (with identity) and $M$ a two-sided ideal of $\Gamma$ and $\Gamma / M$ the quotient algebra.

Definition 2.1. The stability Lie algebra of the chain $\Gamma \supset M \supset 0$ denoted by $S(\Gamma \supset M \supset 0)$ is the set of all derivations of $\Gamma$ which are trivial on $M$ and which induce the trivial derivation on $\Gamma / M . S(T \supset M \supset 0)$ is an abelian subalgebra of the Lie algebra $\mathscr{D}(\Gamma)$ of all derivations of $\Gamma$. Let $A$ be a $K$-projective associative algebra (with identity) and $A$ a two sided $A$-module. Let $(\Sigma): 0 \longrightarrow A \stackrel{\beta}{\longrightarrow} \Gamma$ $\stackrel{a}{\longrightarrow} \Lambda \longrightarrow 0$ be an extension over $\Lambda$ with kernel $A$ (abelian).

Lemma 2.2. There exists a K-isomorphism of $S(\Gamma \supset \beta(A) \supset 0)$ onto $Z^{1}(A, A)$ (the $K$-module of 1-cocycles of $A$ with values in $A$ ).

Proof. Let $\theta: S(\Gamma \supset \beta(A) \supset 0) \rightarrow Z^{1}(\Lambda, A)$ be the mapping defined by the relation $\beta(\theta(s)(\lambda))=s(\gamma), \gamma \in \Gamma, s \in S$, and $\lambda=\alpha(\gamma) \in A$. Then $\theta$ is a $K$-isomorphism.

\section{Coherent pairs of extensions of associative algebras}

Let $\lambda_{1}$ and $\lambda_{2}$ be two $K$-projective associative algebras with identity such that the map $k \rightarrow k \cdot 1$ is a monomorphism of $K$ onto a $K$-direct summand of $A_{i}$, $i=1,2$ and $A$ be a two sided $\left(A_{1}, A_{2}\right)$-bimodule.

Let $\left(\Sigma_{1}\right),\left(\Sigma_{2}\right)$

$$
\begin{aligned}
& \left(\Sigma_{1}\right): 0 \longrightarrow A \stackrel{\beta_{1}}{\longrightarrow} \Gamma_{1} \stackrel{\alpha_{1}}{\longrightarrow} \Lambda_{1} \longrightarrow 0 \\
& \left(\Sigma_{2}\right): 0 \longrightarrow A \stackrel{\beta_{2}}{\longrightarrow} \Gamma_{2} \stackrel{\alpha_{2}}{\longrightarrow} \Lambda_{2} \longrightarrow 0
\end{aligned}
$$


be a pair of extensions over $\Lambda_{1}$ and $\Lambda_{2}$ respectively with kernel $A$ (abelian). Let $L\left(\lambda_{i}\right)$ (resp. $R\left(\lambda_{i}\right)$ ), $\lambda_{i} \in \Lambda_{i}$ denote the operator on $A$ corresponding to $\lambda_{i}$ with respect to the left $\Lambda_{i}$-module (resp. right $\Lambda_{i}$-module) structure of $A$. i.e. $L\left(\lambda_{i}\right) \cdot a=\lambda_{i} \cdot a: R\left(\lambda_{i}\right) \cdot a=a \cdot \lambda_{i}, a \in A, \lambda_{i} \in \Lambda_{i}$.

Definition 3.1. A complementary extending derivation for an element $\lambda_{i} \in \Lambda_{i}$, $i=1,2$ is an extension $\zeta_{i}\left(\lambda_{i}\right)$ of the operator $L\left(\lambda_{i}\right)-R\left(\lambda_{i}\right)$ as a derivation of $\Gamma_{j}, i \neq j$ such that $\zeta_{i}\left(\lambda_{i}\right)$ induces the trivial derivation on $\Lambda_{j}$.

Definition 3.2. A pair $\left(\Sigma_{1}\right),\left(\Sigma_{2}\right)$ of extensions over $\Lambda_{1}$ and $\Lambda_{2}$ respectively with kernel $A$ is called a complementary pair of extensions if, for $i=1,2$ there exists a $K$-homomorphism $\zeta_{i}: \Lambda_{i} \rightarrow \mathscr{D}\left(I^{\prime}\right), i \neq j$, as $K$-modules, such that $\zeta_{i}\left(\lambda_{i}\right)$ is a complementary extending derivation for $\lambda_{i}$ and $\zeta_{i}(1)=0$.

Since $\Lambda_{i}$ is $K$-projective, the exact sequence $\left(\Sigma_{i}\right), i=1,2$ splits as a sequence of $K$-modules. Further, as $K \cdot 1$ is a $K$-direct summand of $\Lambda_{i}$, we can assume that $\Lambda_{i}=K \cdot 1 \oplus \Lambda_{i}^{\prime}$ and the splitting $u_{i}: \Lambda_{i} \rightarrow \Gamma_{i}$ can be chosen such that $u_{i}(1)$ $=1$. Let $\omega_{i}$ be the 2 -cocycle of $\Lambda_{i}$ with values in $A$ associated to the extension ( $\Sigma_{i}$ ) by means of the splitting $u_{i}$. i.e.

$$
u_{i}\left(\lambda_{1}^{(i)}\right) \cdot u_{i}\left(\lambda_{2}^{(i)}\right)-u_{i}\left(\lambda_{1}^{(i)} \cdot \lambda_{2}^{(i)}\right)=\beta_{i}\left(\omega_{i}\left(\lambda_{1}^{(i)}, \lambda_{2}^{(i)}\right)\right), \lambda_{1}^{(i)}, \lambda_{2}^{(i)} \in \Lambda_{i} .
$$

$\omega_{i}$ is then a normalized 2-cocycle and the elements $r_{i}$ of $\Gamma_{i}$ can be expressed uniquely in the form $\gamma_{i}=\beta_{i}\left(a_{i}\right)+u_{i}\left(\lambda_{i}\right), \lambda_{i}=\alpha_{i}\left(\gamma_{i}\right)$ for some $a_{i} \in A$.

Proposirion 3.3. $\left(\Sigma_{1}\right),\left(\Sigma_{2}\right)$ is a complementary pair if and only if there exists a pair of functions $r_{1}, r_{2}$ such that $r_{i} \in \operatorname{Hom}_{K}\left(\widetilde{N}_{1}\left(\Lambda_{1}\right) \otimes \widetilde{N}_{1}\left(\Lambda_{2}\right), A\right)$ satisfying the following conditions:

$$
\begin{aligned}
& \beta_{j}\left(r_{i}\left(\lambda_{1}, \lambda_{2}\right)\right)=\left(\zeta_{i}\left(\lambda_{i}\right)\right)\left(u_{j}\left(\lambda_{j}\right)\right) \\
& \delta_{j} r_{i}=\delta_{i} \omega_{j}, \quad i \neq j, i=1,2 .
\end{aligned}
$$

Proof. Let $\left(\Sigma_{1}\right),\left(\Sigma_{2}\right)$ be a complementary pair. Then there exists a $K$ homomorphism $\left.\zeta_{i}: \Lambda_{i} \rightarrow \mathfrak{D} \cdot \Gamma_{j}\right), i \neq j$ (as $K$-modules) such that $\zeta_{i}\left(\lambda_{i}\right)$ is a complementary extending derivation for $\lambda_{i}, i=1,2$ and $\zeta_{i}(1)=0$. As $\zeta_{i}\left(\lambda_{i}\right)$ induces the trivial derivation on $\Lambda_{j}, i \neq j\left(\zeta_{i}\left(\lambda_{i}\right)\right)\left(\gamma_{j}\right) \in \beta_{j}(A)$ for $\gamma_{j} \in \Gamma_{j}$. In particular for $\lambda_{j} \in \Lambda_{j},\left(\zeta_{i}\left(\lambda_{i}\right)\right)\left(u_{j}\left(\lambda_{j}\right)\right) \in \beta_{j}(A) . \quad\left(u_{j}\right.$ is a splitting of $\left.\left(\Sigma_{j}\right)\right)$. Let us denote by $\beta_{j}\left(r_{i}\left(\lambda_{1}, \lambda_{2}\right)\right)$ the element $\left(\zeta_{i}\left(\lambda_{i}\right)\right)\left(u_{j}\left(\lambda_{j}\right)\right)$. Thus we have function $r_{i}, i=1,2$, $r_{i}: \Lambda_{1} \times \Lambda_{2} \rightarrow A$ such that $\beta_{j}\left(r_{i}\left(\lambda_{1}, \lambda_{2}\right)\right)=\left(\zeta_{i}\left(\lambda_{i}\right)\right)\left(u_{j}\left(\lambda_{j}\right)\right), i \neq j$. Since $\zeta_{i}: \Lambda_{i} \rightarrow \mathscr{D}\left(\Gamma_{j}\right)$ is a $K$-homomorphism and $\zeta_{i}(1)=0$.it follows that $r_{i} \in \operatorname{Hom}_{K}\left(\tilde{N}_{1}\left(\Lambda_{1}\right) \otimes\right.$ 
$\left.\tilde{N}_{1}\left(\Lambda_{2}\right), A\right)$. As $\zeta_{i}\left(\lambda_{i}\right)$ is a derivation of $\Gamma_{j}$ it can be seen that the 2-cocycles $\omega_{i}$ (normalized) corresponding to the extension $\left(\Sigma_{i}\right)$ satisfy the relation (7). Conversely, we define $\zeta_{i}: \Lambda_{i} \rightarrow \mathfrak{D}\left(\Gamma_{j}\right), i \neq j$ as follows :

$$
\begin{aligned}
& \gamma_{j} \in \Gamma_{j}, \gamma_{j}=\beta_{j}\left(a_{j}\right)+u_{j}\left(\lambda_{j}\right), \lambda_{j}=\alpha_{j}\left(\gamma_{j}\right) \\
& \begin{aligned}
\left(\zeta_{i}\left(\lambda_{i}\right)\right)\left(\gamma_{j}\right) & =\left(\zeta_{i}\left(\lambda_{i}\right)\right)\left(\beta_{j}\left(a_{j}\right)\right)+\left(\zeta_{i}\left(\lambda_{i}\right)\right)\left(u_{j}\left(\lambda_{j}\right)\right) \\
& =\beta_{j}\left(\left(L\left(\lambda_{i}\right)-R\left(\lambda_{i}\right)\right)\left(a_{j}\right)+r_{i}\left(\lambda_{1}, \lambda_{2}\right)\right) .
\end{aligned}
\end{aligned}
$$

The relation (7) shows that $\zeta_{i}\left(\lambda_{i}\right)$ is a complementary extending derivation and, as $r_{i} \in \operatorname{Hom}_{K}\left(\tilde{N}_{1}\left(\Lambda_{1}\right) \otimes \tilde{N}_{1}\left(\Lambda_{2}\right), A\right), \zeta_{i}$ is a homomorphism of $K$-modules.

Lemma 3.4. Let $\left(\Sigma_{1}\right),\left(\Sigma_{2}\right)$ be a complementary pair and $u_{i}, u_{i}^{\prime}$ be two splittings of $\left(\Sigma_{i}\right)$ as K-modules such that $u_{i}(1)=u_{i}^{\prime}(1)=1$ and $r_{i}, r_{i}^{\prime}$ be the associated functions. Then there exists $c_{i} \in \operatorname{Hom}_{K}\left(\tilde{N}_{1}\left(\Lambda_{i}\right), A\right)$ such that $r_{i}^{\prime}=r_{i}+\delta_{i} c_{j}, \quad i \neq j$, $i=1,2$.

Lemma 3.5. Let $\left(\Sigma_{1}\right),\left(\Sigma_{2}\right)$ be a complementary pair. Any two complementary extending derivations $\zeta_{i}\left(\lambda_{i}\right), \zeta_{i}^{\prime}\left(\lambda_{i}\right)$ for $\lambda_{i} \in \Lambda_{i}$ differ by an element $s\left(\lambda_{i}\right) \in S\left(\Gamma_{j} \supset\right.$ $\left.\beta_{j}(A) \supset 0\right) \quad i \neq j$ and conversely.

The proofs of these two lemmas are straight forward verifications.

Definition 3.6. A partial cocycle $z$ is an element $z \in \operatorname{Hom}_{K}\left(\widetilde{N}_{1}\left(\Lambda_{1}\right) \otimes \widetilde{N}_{1}\left(\Lambda_{2}\right)\right.$, $A$ ) such that $z$ belongs to $\operatorname{Hom}_{E}\left(\tilde{N}_{1}\left(\Lambda_{j}\right), Z^{1}\left(\Lambda_{i}, A\right)\right.$ ) for $i=1$ or $2, i \neq j$ (In this case, we denote $z$ by $z_{i}$ ).

Proposition 3.7. Let $\left(\Sigma_{1}\right),\left(\Sigma_{2}\right)$ be a complementary pair. Then for (i) different splittings $u_{i}^{\prime}$ of $\left(\Sigma_{i}\right)$ such that $u_{i}^{\prime}(1)=1$ (ii) complementary extending derivations of $\Gamma_{j}$ for elements of $\Lambda_{i}, i \neq j$ for different homomorphisms $\zeta_{i}^{\prime}: \Lambda_{i} \rightarrow$ $\mathfrak{D}\left(\Gamma_{j}\right)$ as K-modules, the sum $r_{1}+r_{2}$ (of the associated functions $r_{i}$ as in Prop. 3.3) gets changed into $r_{1}+r_{2}+z_{1}+z_{2}$ where $z_{i}, i=1,2$ are partial cocycles, $z_{i} \in \mathrm{Hom}_{K}$ $\left(\tilde{N}_{1}\left(\Lambda_{j}\right), Z^{1}\left(\Lambda_{i}, A\right)\right)$.

The proof is a straightforward verification.

Definition 3.8. A complementary pair $\left(\Sigma_{1}\right),\left(\Sigma_{2}\right)$ of extensions over $\Lambda_{1}$ and $\Lambda_{2}$ respectively with kernel $A$ is called a coherent pair if, for some splittings $u_{i}$ of $\left(\Sigma_{i}\right)$ such that $u_{i}(1)=1$ and some $K$-homomorphism $\zeta_{i}: \Lambda_{i} \rightarrow \mathcal{D}\left(\Gamma_{j}\right) i \neq j$ (as $K$-modules) such that $\zeta_{i}(1)=0$ and $\zeta_{i}\left(\lambda_{i}\right)$ is a complementary extending derivation of $\Gamma_{j}$ for $\lambda_{i}$, the sum $r_{1}+r_{2}$ of the associated functions $r_{i}$ (as in Prop. 3,3) 
is equal to the sum $z_{1}+z_{2}$ for some partial cocycles

$$
z_{i} \in \operatorname{Hom}_{k}\left(\tilde{N}_{1}\left(\Lambda_{j}\right), Z^{1}\left(\Lambda_{i}, A\right)\right), i \neq j, i=1,2 .
$$

From Prop. 3.7, we see that this property of being coherent depends only on the extensions $\left(\Sigma_{1}\right),\left(\Sigma_{2}\right)$. We give a criterion for coherence in the following.

PROPOsition 3.9. A complementary pair $\left(\Sigma_{1}\right),\left(\Sigma_{2}\right)$ is a coherent pair, if and only if, for some choice of splittings $u_{i}$ of $\left(\Sigma_{i}\right)$ with $u_{i}(1)=1$ and K-homomorphism $\zeta_{i}: \Lambda_{i} \rightarrow \mathfrak{D}\left(\Gamma_{j}\right), i \neq j$ (as K-modules) for $i=1,2$ such that $\zeta_{i}(1)=0$ and $\zeta_{i}\left(\lambda_{i}\right)$ is complementary extending derivation for $\lambda_{i}$, the sum $\rho_{1}+\rho_{2}$ of the associated functions $\rho_{i}$ (as given by Prop. 3.3) is 0 .

Proof. Let $\left(\Sigma_{1}\right),\left(\Sigma_{2}\right)$ be a coherent pair. Then for some splitting $u_{i}$ of $\left(\Sigma_{i}\right)$ such that $u_{i}(1)=1$ and homomorphism $\zeta_{i}: \Lambda_{i} \rightarrow \mathscr{D}\left(\Gamma_{j}\right), i \neq j, i=1,2$ the sum $r_{1}+r_{2}$ of the associated functions $r_{i}$ is equal to the sum $z_{1}+z_{2}, z_{i}$ being partial cocycle belonging to $\operatorname{Hom}_{K}\left(\tilde{N}_{1}\left(\Lambda_{j}\right), Z^{1}\left(\Lambda_{i}, A\right)\right), i=1,2$ i.e. $r_{1}+r_{2}=z_{1}+z_{2}$. With the same splittings $u_{i}$ but with the homomorphism $\zeta_{i}^{\prime}: \Lambda_{i} \rightarrow \mathscr{D}\left(\Gamma_{j}\right)$, $\zeta_{i}^{\prime}=\zeta_{i}+\theta_{j}^{-1}\left(-z_{j}\right)\left(\theta_{j}\right.$ is the $K$-isomorphism of $S\left(\Gamma_{j} \supset \beta_{j}(A) \supset 0\right)$ onto $Z^{1}\left(\Lambda_{j}, A\right)$ of Lemma 2.21, we see that the sum $\rho_{1}+\rho_{2}$ of the associated functions $\rho_{i}$ is equal to $r_{1}+r_{2}-z_{1}-z_{2}=0$. Conversely if $\rho_{1}+\rho_{2}=0$ with $z_{1}=z_{2}=0$ we see that the pair $\left(\Sigma_{1}\right),\left(\Sigma_{2}\right)$ is coherent.

\section{An example of a coherent pair}

Let $\Lambda=\Lambda_{1} \otimes \Lambda_{2}$ (with the same assumptions on $\Lambda_{i}$ and $K$, as earlier). Then $A$ is a two sided $A$-module. Let $(\Sigma): 0 \longrightarrow A \stackrel{\beta}{\longrightarrow} I \stackrel{\alpha}{\longrightarrow} \Lambda \longrightarrow 0$ be an extension over $A$ with kernel $A$ (abelian). Further let $\Gamma_{i}=\alpha^{-1}\left(\Lambda_{i}\right), i=1,2$ ( $\Lambda_{i}$ is identified as a sub-algebra of $\Lambda)$. Then we have a pair $\left(\Sigma_{1}\right),\left(\Sigma_{2}\right)$ of extensions, $\left(\Sigma_{i}\right): 0 \longrightarrow A \stackrel{\beta_{i}}{\longrightarrow} \Gamma_{i} \stackrel{\alpha_{i}}{\longrightarrow} \Lambda_{i} \longrightarrow 0, \alpha_{i}=\alpha \mid \Gamma_{i}$ and $\beta_{i}=\beta$. Let $u_{i}: \Lambda_{i} \rightarrow \Gamma_{i}$ be a splitting of $\left(\Sigma_{i}\right)$ as $K$-modules such that $u_{i}(1)=1$. We shall define a complementary extending derivation $\zeta_{i}\left(\lambda_{i}\right)$ for $\lambda_{i}, i=1,2$ as follows:

$$
\begin{gathered}
\left(\zeta_{i}\left(\lambda_{i}\right)\right)\left(\gamma_{j}\right)=\left[u_{i}\left(\lambda_{i}\right), \gamma_{j}\right]=u_{i}\left(\lambda_{i}\right) \cdot \gamma_{j}-\gamma_{j} \cdot u_{i}\left(\lambda_{i}\right) \\
i \neq j, i=1,2: \gamma_{j} \in \Gamma_{j} \text { and } \lambda_{i} \in \Lambda_{i} .
\end{gathered}
$$

Then $\zeta_{i}\left(\lambda_{i}\right)$ is a derivation of $\Gamma_{j}, i \neq j, \zeta_{i}(1)=0$ and induces trivial derivation on $\Lambda_{j}$ and $\zeta_{i}: \Lambda_{i} \rightarrow \mathfrak{D}\left(\Gamma_{j}\right)$ is a $K$-homomorphism. Thus $\left(\Sigma_{1}\right),\left(\Sigma_{2}\right)$ is a complementary pair. $\beta_{j}\left(r_{i}\left(\lambda_{1}, \lambda_{2}\right)\right)=\left(\zeta_{i}\left(\lambda_{i}\right)\right)\left(u_{j}\left(\lambda_{j}\right)\right)=\left[u_{j}\left(\lambda_{i}\right), u_{j}\left(\lambda_{j}\right)\right], i \neq j$. Hence $r_{1}+r_{2}=0$ i.e. $\left(\Sigma_{1}\right),\left(\Sigma_{2}\right)$ is a coherent pair. 
Proposition 3.10. Let $\left(\Sigma_{1}\right),\left(\Sigma_{2}\right)$

$$
\begin{aligned}
& \left(\Sigma_{1}\right): 0 \longrightarrow A \stackrel{\beta_{1}}{\longrightarrow} \Gamma_{1} \stackrel{\alpha_{1}}{\longrightarrow} \Lambda_{1} \longrightarrow 0 \\
& \left(\Sigma_{2}\right): 0 \longrightarrow A \stackrel{\beta_{2}}{\longrightarrow} \Gamma_{2} \stackrel{\alpha_{2}}{\longrightarrow} \Lambda_{2} \longrightarrow 0
\end{aligned}
$$

be a coherent pair of extensions (with the same assumptions on $\Lambda_{i}$ and $K$, as earlier). Then (i) there exists an extension $(\Sigma)$

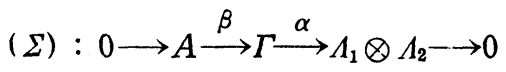

over $\Lambda_{1} \otimes \Lambda_{2}$ with kernel $A$

(ii) there exists operator $K$-isomorphisms $\varphi_{i}: \alpha^{-1}\left(\Lambda_{i}\right) \rightarrow I_{i}, i=1,2$ (with respect to operators from $\Lambda_{j}, i \neq j$ and

(iii) $\alpha_{i} \cdot \varphi_{i}=\alpha \mid \alpha^{-1}\left(\Lambda_{i}\right), i=1,2$.

Proof. Let $\left(\Sigma_{i}\right): 0 \longrightarrow A \stackrel{\beta_{i}}{\longrightarrow} \Gamma_{i} \stackrel{\alpha_{i}}{\longrightarrow} \Lambda_{i} \longrightarrow 0$ be a coherent pair. Let us choose (i) splitting $u_{i}: \Lambda_{i} \rightarrow \Gamma_{i}$ such that $u_{i}(1)=1$ with $\omega_{i}$ as associated 2-cocycles (normalized) and (2) $K$-homomorphism $\zeta_{i}: \Lambda_{i} \rightarrow \mathscr{D}\left(\Gamma_{j}\right), i \neq j$ so that the sum $r_{1}+r_{2}=0$ (of the associated functions $r_{i}$ (Prop. 3.9)). Let $r=r_{1}=-r_{2}$. Then the 2-bicochain $\omega_{1}+r+\omega_{2}$ is a 2-bicocycle and let $\omega=\xi^{*}\left(\omega_{1}+r+\omega_{2}\right)$ ( $\xi^{*}$ given by (4)). Then $\omega$ is a 2-cocycle (normalized) of $\Lambda_{1} \otimes \Lambda_{2}$ with values in $A$. Let $\Gamma=A \oplus\left(\Lambda_{1} \otimes \Lambda_{2}\right)$ (direct sum as $K$-modules). We shall, as usual, denote by the pairs $\left(a, \Sigma_{i} \lambda_{1 i} \otimes \lambda_{2 i}\right)$ the elements of $\Gamma, a \in A, \lambda_{1 i} \in \Lambda_{1}, \lambda_{2 i} \in \Lambda_{2}$. Let $\beta: A \rightarrow \Gamma, \alpha: \Gamma \rightarrow \Lambda_{1} \otimes \Lambda_{2}$ be defined by $\beta(a)=(a, 0)$ and $\alpha\left(\left(a, \sum \lambda_{1} i \otimes \lambda_{2} i\right)\right)=$ $\sum \lambda_{1 i} \otimes \lambda_{2 i}$. We define a multiplication in $\Gamma$, as usual, by means of the 2-cocycle $\omega$.

$$
\begin{aligned}
& \left(a_{1}, \lambda_{11} \otimes \lambda_{21}\right) \cdot\left(a_{2}, \lambda_{12} \otimes \lambda_{22}\right) \\
& =\left(a_{1} \cdot \lambda_{12} \cdot \lambda_{22}+\lambda_{11} \cdot \lambda_{12} \cdot a_{2}+\omega_{1}\left(\lambda_{11}, \lambda_{12}\right) \lambda_{21} \cdot \lambda_{22}\right. \\
& \left.\quad+\lambda_{21} r\left(\lambda_{11}, \lambda_{22}\right) \lambda_{12}+\lambda_{11} \cdot \lambda_{12} \omega_{2}\left(\lambda_{21}, \lambda_{22}\right), \lambda_{11} \cdot \lambda_{12} \otimes \lambda_{21} \cdot \lambda_{22}\right)
\end{aligned}
$$

(from (4)). Then $\Gamma$ is an associative $K$-algebra and $(\Sigma): 0 \rightarrow A \stackrel{\beta}{\longrightarrow} \Gamma \stackrel{\alpha}{\longrightarrow} \Lambda_{1} \otimes$ $\Lambda_{2} \rightarrow 0$ is an extension over $\Lambda_{1} \otimes \Lambda_{2}$ with kernel $A$. As in the example given above, this gives rise to a coherent pair $\left(\Sigma_{1}^{\prime}\right),\left(\Sigma_{2}^{\prime}\right)$ of extensions over $\Lambda_{1}$ and $\Lambda_{2}$ respectively with kernel $A$ where

$$
\left(\Sigma_{i}^{\prime}\right): 0 \longrightarrow A \stackrel{\beta_{i}^{\prime}}{\longrightarrow} \alpha^{-1}\left(\Lambda_{i}\right) \stackrel{\alpha_{i}^{\prime}}{\longrightarrow} \Lambda_{i} \longrightarrow 0
$$

where $\alpha_{i}^{\prime}=\alpha \mid \alpha^{-1}\left(\Lambda_{i}\right)$ and $\beta_{i}^{\prime}=\beta$. Let us define the mapping $\varphi_{i}: \alpha^{-1}\left(\Lambda_{i}\right) \rightarrow \Gamma_{i}$ as follows. 
Case $i=1: \varphi\left(\left(a, \lambda_{1} \otimes 1\right)\right)=\beta_{1}(a)+u_{1}\left(\lambda_{1}\right)$

Case $i=2: \varphi_{2}\left(\left(a, 1 \otimes \lambda_{2}\right)\right)=\beta_{2}(a)+u_{2}\left(\lambda_{2}\right)$.

Then $\varphi_{i}, i=1,2$ is an isomorphism of $K$-algebras. We shall verify here only that the multiplication is preserved and this is operator isomorphism.

Case $i=1, j=2$ :

$$
\begin{aligned}
& \varphi_{1}\left(\left(a, \lambda_{11} \otimes 1\right) \cdot\left(a_{2}, \lambda_{12} \otimes 1\right)\right) \\
= & \beta_{1}\left(a_{1} \cdot \lambda_{12}+\lambda_{11} \cdot a_{2}+\omega_{1}\left(\lambda_{11}, \lambda_{12}\right)\right)+u_{1}\left(\lambda_{11} \cdot \lambda_{12}\right) \\
= & \varphi_{1}\left(\left(a_{1}, \lambda_{11} \otimes 1\right)\right) \cdot \varphi_{1}\left(\left(a_{2}, \lambda_{12} \otimes 1\right)\right)
\end{aligned}
$$

Let $\lambda_{2} \in \Lambda_{2}$ :

$$
\begin{aligned}
& \varphi_{1}\left(\left[\left(0,1 \otimes \lambda_{2}\right),\left(a, \lambda_{1} \otimes 1\right)\right]\right) \\
= & \varphi_{1}\left(\left(L\left(\lambda_{2}\right)-R\left(\lambda_{2}\right)\right)(a)-r\left(\lambda_{1}, \lambda_{2}\right), 0\right) \\
= & \beta_{1}\left(\left(L\left(\lambda_{2}\right)-R\left(\lambda_{2}\right)\right)(a)-r\left(\lambda_{1}, \lambda_{2}\right)\right) \\
= & \zeta_{2}\left(\lambda_{2}\right) \cdot \varphi_{1}\left(\left(a, \lambda_{1} \otimes 1\right)\right) .
\end{aligned}
$$

i.e. $\varphi_{1}: \alpha^{-1}\left(\Lambda_{1}\right) \rightarrow \Gamma_{1}$ is operator isomorphism with operators from $\Lambda_{2}$. Similarly for $i=2$ we show $\varphi_{2}$ is operator isomorphism of $\alpha^{-1}\left(\Lambda_{2}\right)$ onto $I_{2}$. Further $\left(\alpha_{1} \cdot \varphi_{1}\right)\left(\left(a, \lambda_{1} \otimes 1\right)\right)=\lambda_{1}=\alpha\left(\left(a, \lambda_{1} \otimes 1\right)\right)$ i.e. $\alpha_{1} \cdot \varphi_{1}=\alpha \mid \alpha^{-1}\left(\lambda_{1}\right)$. Similarly $\alpha_{2} \cdot \varphi_{2}$ $=\alpha \mid \alpha^{-i}\left(\Lambda_{2}\right)$. We summarize together the Prop. 3.10 and the example given earlier in

THEOREM 3.11. Let $(\Sigma): 0 \longrightarrow A \stackrel{\beta}{\longrightarrow} \Gamma \stackrel{\alpha}{\longrightarrow} \Lambda \longrightarrow 0$ be an extension over $\Lambda=\Lambda_{1} \otimes \Lambda_{2}$ with kernel $A, \Lambda_{i}$ being $K$-projective associative algebra with identity such that the map $k \rightarrow k \cdot 1$ is a monomorphism of $K$ onto a $K$-direct summand of $\Lambda_{i}, i=1,2$ and $A$ a two sided $\left(\Lambda_{1}, \Lambda_{2}\right)$-bimodule. Then $\left(\Sigma_{i}^{\prime}\right): 0 \rightarrow A \rightarrow \alpha^{-1}\left(\Lambda_{i}\right) \rightarrow \Lambda_{i} \rightarrow 0 i=1,2$ is a coherent pair of extensions over $\Lambda_{i}$ with kernel $A$, the complementary extending derivations on $\alpha^{-1}\left(\Lambda_{j}\right)$ for $\lambda_{i} \in \Lambda_{i}, i \neq j$ being given by inner derivation of coset representatives $u_{i}\left(\lambda_{i}\right)$ of elements of $\Lambda_{i}$ in $\alpha^{-1}\left(\Lambda_{i}\right), u_{i}: \Lambda_{i} \rightarrow \alpha^{-1}\left(\Lambda_{i}\right)$ being a splitting of $\left(\Sigma_{i}^{\prime}\right)$ as $K$-modules with $u_{i}(1)=1$. Conversely if $\left(\Sigma_{i}\right): 0 \longrightarrow A \stackrel{\beta_{i}}{\longrightarrow} \Gamma_{i} \stackrel{\alpha_{i}}{\longrightarrow} \Lambda_{i} \longrightarrow 0$ $(i=1,2)$ is a coherent pair of extensions over $\Lambda_{i}$ with kernel $A$ then (1) there exists an extension $(\Sigma): 0 \longrightarrow A \stackrel{\beta}{\longrightarrow} \Gamma \stackrel{\alpha}{\longrightarrow} \Lambda_{1} \otimes \Lambda_{2} \longrightarrow 0$ over $\Lambda_{1} \otimes \Lambda_{2}$ with kernel $A$ (2) there exists a pair of operator isomorphisms $\varphi_{i}$ from $\alpha^{-1}\left(\Lambda_{i}\right)$ onto $\Gamma_{i}, i=1,2$ (as $K$-algebras) and (3) $\alpha_{i} \cdot \varphi_{i}=\alpha \mid \alpha^{-1}\left(\Lambda_{i}\right), i=1,2$.

Let $\mathscr{C}\left(\Lambda_{1}, \Lambda_{2} ; A\right)$ denote the set of all coherent pairs of equivalence classes 
of extensions over $\Lambda_{1}$ and $\Lambda_{2}$ with kernel $A$ (with the same assumptions on $\Lambda_{i}$, $A$ and $K$, as earlier). A coherent pair $\left(\Sigma_{1}\right),\left(\Sigma_{2}\right)$ of extensions determines at least one quadruple $\left\{\omega_{1}, r_{1}, r_{2}, \omega_{2}\right\}$ where $\omega_{i} \in Z^{2}\left(\Lambda_{i}, A\right)$ (normalized 2-cocycle) and $r_{i} \in \operatorname{Hom}_{K}\left(\tilde{N}_{1}\left(\Lambda_{1}\right) \otimes \tilde{N}_{1}\left(\Lambda_{2}\right), A\right)$ such that (1) $\delta_{i} \omega_{j}=\delta_{j} r_{i}, i \neq j, i=1,2$ and (2) $r_{1}+r_{2}=z_{1}+z_{2}$ where $z_{i}$ is a partial cocycle, $z_{i} \in \operatorname{Hom}_{\kappa}\left(\tilde{N}_{1}\left(\Lambda_{j}\right), Z^{1}\left(\Lambda_{i}, A\right)\right.$ ), $i \neq j$ we shall call such a quadruple a standard quadruple. Conversely, any standard quadruple $\left\{\omega_{1}, r_{1}, r_{2}, \omega_{2}\right\}$ determines a coherent pair of extensions.

Definition 3.12. Two standard quadruples $\left\{\omega_{1}, r_{1}, r_{2}, \omega_{2}\right\}\left\{\omega_{1}^{\prime}, r_{1}^{\prime}, r_{2}^{\prime}, \omega_{2}^{\prime}\right\}$ are called equivalent if (1) there exists a 1-cochain $c_{i}$ (normalized) such that $\omega_{i}^{\prime}-\omega_{i}=\delta_{i} c_{i}$ for $i=1,2$ and (2) there exists partial cocycle $z_{i}$ such that $r_{i}^{\prime}-r_{i}=\delta_{i} c_{j}+z_{j} . \quad$ A standard quadruple of the form $\left\{\delta_{1} c_{1}, \delta_{1} c_{2}+z_{2}, \delta_{2} c_{1}+z_{1}, \delta_{2} c_{2}\right\}$ is called a trivial quadruple.

Let $\mathscr{S}\left(\Lambda_{1}, \Lambda_{2} ; A\right)$ denote the set of all equivalence classes of standard quadruples. Then $\mathscr{S}\left(\Lambda_{1}, \Lambda_{2} ; A\right)$ is a $K$-module under componentwise addition and scalar multiplication of representatives. It is also clear that there exists a bijection of $\mathscr{S}\left(\Lambda_{1}, \Lambda_{2} ; A\right)$ onto $\mathscr{E}\left(\Lambda_{1}, \Lambda_{2} ; A\right)$. Hereafter we shall identify $\mathscr{C}$ with $\mathscr{S}$. $\mathscr{C}$ is not empty because the pair $\left(\Sigma_{1}\right),\left(\Sigma_{2}\right)$ where $\left(\Sigma_{i}\right)$ is the inessential extension over $\Lambda_{i}$ with kernel $A$, is a coherent pair. Let us now define the following maps

$$
\begin{aligned}
& \Omega_{i}: H^{2}\left(\Lambda_{1}, \Lambda_{2} ; A\right) \rightarrow H^{2}\left(\Lambda_{i}, A\right), i=1,2 \\
& \Omega: H^{2}\left(\Lambda_{1}, \Lambda_{2} ; A\right) \rightarrow H^{2}\left(\Lambda_{1}, A\right) \oplus H^{2}\left(\Lambda_{2}, A\right) \\
& \Pi: H^{2}\left(\Lambda_{1}, \Lambda_{2} ; A\right) \rightarrow \mathscr{S}\left(\Lambda_{1}, \Lambda_{2} ; A\right) \\
& \Delta: \mathscr{S}\left(\Lambda_{1}, \Lambda_{2} ; A\right) \rightarrow H^{2}\left(\Lambda_{1}, A\right) \oplus H^{2}\left(\Lambda_{2}, A\right) .
\end{aligned}
$$

Let $\omega_{1}+r+\omega_{2}$ be a representative 2-bicocycle of an element of $H^{2}\left(\Lambda_{1}, \Lambda_{2} ; A\right)$

$$
\begin{aligned}
& \Omega_{i}\left(\omega_{1}+r+\omega_{2}\right)=\text { the cohomology class of } \omega_{i} \text { in } H^{2}\left(\Lambda_{i}, A\right) \\
& \Omega\left(\omega_{1}+r+\omega_{2}\right)=\Omega_{1}\left(\omega_{1}+r+\omega_{2}\right)+\Omega_{2}\left(\omega_{1}+r+\omega_{2}\right) . \\
& \Pi\left(\omega_{1}+r+\omega_{2}\right)=\text { equivalence class of the standard quadruple }\left\{\omega_{1}, r,-r, \omega_{2}\right\} \\
& \left.\Lambda\left(\left\{\omega_{1}, r_{1}, r_{2}, \omega_{2}\right\}\right)=\text { (class of } \omega_{1}, \text { class of } \omega_{2}\right) .
\end{aligned}
$$

These are well defined $K$-homomorphisms. Let $\Omega_{i}$ be the kernel of $\Omega_{i}$ and $R$ the kernel of $\Omega$. Then $\Omega=\Omega_{1} \cap \Omega_{2}$. 
Proposition 3.13. $\Re$ consists of the classes of elements of the form $(0, r, 0)$ where $r \in \operatorname{Hom}_{K}\left(\tilde{N}_{1}\left(\Lambda_{1}\right) \otimes \tilde{N}_{1}\left(\Lambda_{2}\right), A\right)$ such that $\delta_{i} r=0, i=1,2$.

The proof is straightforward verification.

Proposition 3.14. II is an epimorphism.

Proof: Let $\left\{\omega_{1}, r_{1}, r_{2}, \omega_{2}\right\}$ be a representative of an element in $\mathcal{S}$. As this standard quadruple corresponds to a coherent pair of extensions, we can assume that $r_{1}=r=-r_{2}$. Then $\omega_{1}+r+\omega_{2}$ is a 2-bicocycle and the image under $I I$ of the bicohomology class of $\omega_{1}+r+\omega_{2}$ is the element in $\mathscr{S}$ which we started with.

Proposition 3.15. $\Delta$ is a monomorphism.

Proof. Enough to verify that kernel $\Delta$ is 0 . Let $\left\{\omega_{1}, r_{1}, r_{2}, \omega_{2}\right\}$ be a representative of an element in Ker $\Delta$. Then

$$
\Delta\left(\left\{\omega_{1}, r_{1}, r_{2}, \omega_{2}\right\}\right)=0 \in H^{2}\left(\Lambda_{1}, A\right) \oplus H^{2}\left(\Lambda_{2}, A\right)
$$

i.e. there exists a 1 -cochain $c_{i} \in \operatorname{Hom}_{K}\left(\tilde{N}_{1}\left(\Lambda_{i}\right), A\right)$ such that $\omega_{i}=\delta_{i} c_{i}, i=1,2$. Hence the equivalence class of $\left\{\omega_{1}, r_{1}, r_{2}, \omega_{2}\right\}$ corresponds to the coherent pair of inessential extensions and this is the zero of $\mathcal{S}$ i.e. Ker $\Delta=0$.

Proposition 3.16. The kernel of $I I$ is $\Omega$ (the kernel of $\Omega$ ).

Proof. Let $\omega_{1}+r+\omega_{2}$ be a representative of an element in $\operatorname{Ker} \Pi$. Then $\Pi\left(\omega_{1}+r+\omega_{2}\right)=$ class of $\left\{\omega_{1}, r,-r, \omega_{2}\right\}=0$ in $\mathscr{S} .\left\{\omega_{1}, r,-r, \omega_{2}\right\}$ is equivalent to a quadruple $\left\{\delta_{1} c_{1}, \delta_{1} c_{2}+z_{2}, \delta_{2} c_{1}+z_{1}, \delta_{2} c_{2}\right\}$ for a (normalized) 1-chain $c_{i}, i=1,2$ and partial cocycle $z_{i}, i=1,2$. Thus $\omega_{i}=\delta_{i} c_{i}$ and $r=r_{1}=-r_{2}=\delta_{1} c_{2}+z_{2}=$ $-\delta_{2} c_{1}-z_{1}$. Hence $\left(\omega_{1}+r+\omega_{2}\right)$ is bicohomologous to $\left(0, r^{\prime}, 0\right)$ where $r^{\prime}=\delta_{2} c_{1}+z_{2}$ $=-\delta_{1} c_{2}-z_{1}$.

$\delta_{1} r^{\prime}=\delta_{2} r^{\prime}=0$ i.e. Ker $I \subset \subset \Omega$. Let $\omega_{1}+r+\omega_{2}$ be a representative of an element in $\Re$. Then $\omega_{1}+r+\omega_{2}$ is bicohomologous to a bicocycle of the form $\left(0, r^{\prime}, 0\right)$ where $\delta_{i} r^{\prime}=0, i=1,2$. Then $\Pi\left(\omega_{1}+r+\omega_{2}\right)=\Pi$ (class of $\left.\left(0, r^{\prime}, 0\right)\right)=$ class of $\left\{0, r^{\prime},-r^{\prime}, 0\right\}=0 \in \mathscr{S}$ i.e. $\Re \subset \operatorname{Ker} \Pi$. Hence $\operatorname{Ker} \Pi=\Re$.

We summarize these together in the following:

THEOREM 3.17. Let $\Lambda_{1}$ and $\Lambda_{2}$ be two associative $K$-algebras (with all assumptions as earlier) and let $A$ be a two sided $\left(\Lambda_{1}, \Lambda_{2}\right)$-bimodule. Then we have the following commutative diagram in which rows and columns are exact: 


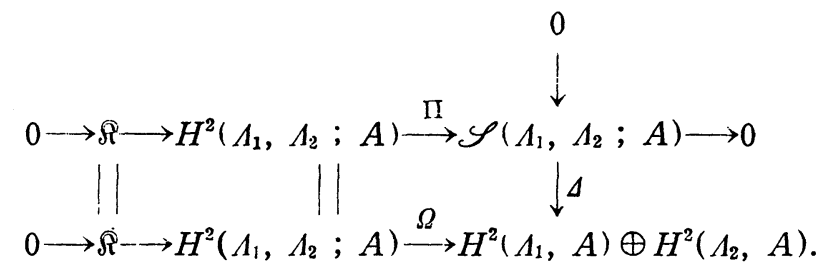

REFERENCES

[1] H. Cartan and S. Eilenberg: HOMOLOGICAL ALGEBRA, Princeton University Press, 1956.

[2] F. Haimo and S. Maclane: The Cohomology theory of a pair of groups, Ill. J. Math., V. 5 (1961), 45-60.

National College,

TIRUCHIRAPALLI-1 (Madras State, INDIA.) 